\title{
Green hydrogen separation from nitrogen by mixed matrix membranes consisting of nanosized sodalite crystals
}

Ge Yang ${ }^{[\mathrm{a}]}$, Hailing Guo*[a] ${ }^{*}$ Zixi Kang ${ }^{[\mathrm{b}]}$, Lei Zhao ${ }^{[\mathrm{a}]}$, Shou Feng ${ }^{[\mathrm{b}]}$, Feng Jiao ${ }^{[\mathrm{a}]}$, Svetlana Mintova ${ }^{*[a, c]}$

Petroleum Corp. (CNPC) China University of Petroleum (East China), Qingdao 266555, People's Republic of China.

b College of Science, China University of Petroleum (East China), Qingdao, Shandong 266580, People's Republic of China.

c Laboratoire Catalyse et Spectrochimie (LCS), Normandie University, ENSICAEN, UNICAEN, CNRS, 6 boulevard du Marechal Juin, 14050 Caen, France

* Corresponding author: Hailing Guo, Svetlana Mintova, fax number: + 33(0)231452821 and email: mintova@ensicaen.fr

Keywords: Mixed matrix membranes $\bullet$ Sodalite $\bullet$ Gas separation $\bullet$ Hydrogen $\bullet$ Nitrogen Stability 
Abstract: Nanosized sodalite crystals (Nano-SOD) are used as active filler to prepare mixed matrix membranes (MMMs) for promoting the $\mathrm{H}_{2} / \mathrm{N}_{2}$ gas separation performance. The Nano-SOD with extremely small crystallites $(40-50 \mathrm{~nm})$ are synthesized from a colloidal suspension free of organic structural directing agent, and uniformly dispersed in the Polyetherimide (PEI) matrix. The Nano-SOD filler with a proper aperture size $(2.8 \AA)$ allow only hydrogen molecules passing through and rejecting the nitrogen thus improving the selectivity of the membranes. The high dispersion of nanosized sodalite crystals in the polymer matrix and the proper interactions between the inorganic and organic phases greatly improve the membrane separation performance and minimize interfacial holes. The MMMs showed a high $\mathrm{H}_{2}$ permeability $\left(\sim 7155.1\right.$ Barrer at $25^{\circ} \mathrm{C}$ under atmospheric pressure) and ideal $\mathrm{H}_{2} / \mathrm{N}_{2}$ selectivity factor of $\sim 16.9$ in a single gas test. Moreover, in a gas mixture $\left(\mathrm{H}_{2} / \mathrm{N}_{2}, 25-100^{\circ} \mathrm{C}\right)$, the selectivity factor increased significantly to $\sim 30.9$. The high stability of the MMMs consisting of highly dispersed nanosized SOD crystals in PEI matrix for $\mathrm{H}_{2} / \mathrm{N}_{2}$ separation (6 weeks continuous test), rendered them as important materials for ammonia synthesis application that requires and also releases a large amount of hydrogen.

\section{Introduction}

Ammonia is one of the most important industry material, which has an over 200 million tons annual production. ${ }^{[1]}$ The ammonia synthesis is a high energy consumption industry, which consumes nearly $1.4 \%$ of all global energy, ${ }^{[2]}$ mainly consumed by feed gas hydrogen production. Nevertheless synthetic ammonia requires vast hydrogen, also releases large amounts of hydrogen. Its purge gas (around 40 billion $\mathrm{Nm}^{3}$ annual) mainly contains $60-70 \%$ hydrogen and $20-25 \%$ nitrogen, leading to a serious air pollution and around 26 billion $\mathrm{Nm}^{3}$ hydrogen waste. Therefore, recovering and reusing hydrogen from the purge gas and for synthetic ammonia process is environmental and economic. $\mathrm{H}_{2} / \mathrm{N}_{2}$ is one of the most difficult gas pairs to be separated, due to the following reasons: (1) similar molecular size $\left(\mathrm{H}_{2}\right.$ of $2.89 \AA$ and $\mathrm{N}_{2}$ of $\left.3.64 \AA\right),{ }^{[3]}$ resulting in molecular sieving 
difficulty; (2) similar quadrupole moment (quadrupole moments of $\mathrm{H}_{2}(0)$ and $\mathrm{N}_{2}\left(4.7 \times 10^{-40}\right.$ $\left.\left.\mathrm{Cm}^{2}\right)\right),{ }^{[4-6]}$ leading to similar sorption ability; and (3) relatively low Knudsen selectivity, which for $\mathrm{H}_{2} / \mathrm{N}_{2}$ is only $3.74 .^{[7,8]}$ Thus, the separation of hydrogen from nitrogen requires more attentions.

For gas separation, membrane technology is widely considered together with other methods including pressure swing adsorption (PSA) and cryogenic distillation. The advantages of membranes are low energy consumption, low investments and operation cost, small footprint, easy operation and being environmentally benign. ${ }^{[9-11]}$ Among various membrane materials, the polymeric membranes have reasonably low cost, easy processing and thus widely used in industries. ${ }^{[12]}$ However their performance are limited by a "trade-off" phenomenon between gas selectivity and permeability. This phenomenon is described by Robeson using an upper bound correlation between permeability and selectivity for specific gas pair, which has been used to evaluate polymeric or polymer mix matrix membrane. ${ }^{[13]}$ The metallic membranes, especially Pd membranes, have an excellent $\mathrm{H}_{2}$ permeability and high $\mathrm{H}_{2}$ purity but suffer from hydrogen embrittlement below $300^{\circ} \mathrm{C}$. This implies that high temperature is required which is connected with high-energy process. ${ }^{[10,14]}$ Inorganic membranes using zeolite and MOFs materials have acted as potential separators due to their unique properties, including uniform pores, framework structures, molecular sieving and reasonable stability. ${ }^{[15,16]}$ They exhibit super permselectivity at ambient conditions. While their difficulties in scale up and easy damage limit further industrial application. With the aim of enhancing membrane separation performance, mixed matrix membranes (MMMs) are attracted as they combine advantages of large scale applications of polymeric membranes and high separation performance of inorganic particles (fillers), which is proposed as an effective mean for polymer matrix membranes to break the "trade-off"[17] and obtain high permeability. ${ }^{[18,19]}$ 
Mixed matrix membranes (MMMs) consisting of Polyethersulfone (PES) and zeolites (13X and 4A) were the first attempt for hydrogen separation. ${ }^{[20]}$ The highest $\mathrm{H}_{2}$ permeability obtained was for PES with $50 \%$ 4A zeolite, which was 14.1 Barrer at $25^{\circ} \mathrm{C}$, which was twice higher than for the pure PES membrane's permeability. The ideal selectivity of $\mathrm{H}_{2} / \mathrm{N}_{2}$ increased from 46 to 56 indicating the selectivity promoted by zeolite filler. Chaidou et al. prepared MMMs consisting of Polyimide (PI) and zeolites (13X, 4A and ZSM-5), and they were used in selected gas mixtures of $\mathrm{He}, \mathrm{H}_{2}, \mathrm{CO}_{2}$ and $\mathrm{N}_{2}$ for systematic research. ${ }^{[21]}$ Compared with the PI membrane, the maximal permeability of the PI/13X membrane increased by $178 \%$ (26.34 Barrer), while the $\mathrm{H}_{2} / \mathrm{N}_{2}, \mathrm{H}_{2} / \mathrm{CO}_{2}$ and $\mathrm{He} / \mathrm{N}_{2}$ selectivity of all MMMs with high zeolite loading decreased (up to $-24.1 \%$ ). Peydayesh et al. fabricated MMMs containing DDR type zeolite (pores of $3.6 \times 4.4 \AA$ ) with polyimide for $\mathrm{H}_{2} / \mathrm{CH}_{4}$ separation. ${ }^{[22]}$ These membranes achieved ideal selectivity of 375 and $\mathrm{H}_{2}$ permeability of 34.9 Barrer. Many micronsized zeolites including $13 \mathrm{X},{ }^{[20,21,23]} 4 \mathrm{~A},{ }^{[21,23,24]} \mathrm{ZSM}-5,{ }^{[21]}$ silicate-1, ${ }^{[25]}$ and mesoporous SBA-15 $5^{[26]}$ were used for preparation of membranes, however none of the above zeolites have appropriate pore opening for selective separation of $\mathrm{H}_{2}$. Additionally, the low external surface area and less accessible apertures of micronsized zeolite crystals cause high diffusion resistance. More importantly, the weak adhesion between the polymer and zeolite crystals causes a large number of uncontrollable voids, leading to low selectivity due to the polymer-zeolite interface. Therefore, to reduce or even eliminate interface voids or pore blocking, coupling agents or compatibilizers were used. Duval et al. modified silicalite- 1 by sliane-coupling agents to promote the adhesion between the polymer and zeolite crystals. ${ }^{[26]}$ The modification decreased the permeability but not affecting the selectivity of the membranes. Khan et al. studied the effect of coupling agent aminopropyltrimethoxysilane (APTMS) in Polusulfone (PSF)/zeolite 3A MMMs for $\mathrm{H}_{2} / \mathrm{CO}_{2}$ separation. ${ }^{[27]}$ The selectivity improved from 1.7 to 3.57 with a five-fold permeability reduction. Yong et al. introduced 2, 4, 6-triaminopyrimidine (TAP) as a compatibilizer into PI/zeolite MMMs. ${ }^{[23]}$ The $\mathrm{CO}_{2} / \mathrm{N}_{2}$ and $\mathrm{O}_{2} / \mathrm{N}_{2}$ selectivity of MMMs with TAP 
increased almost five time compared with the MMMs without compatibilizer. However the gas permeability for all decreased greatly which was mainly attributable to the blocked micropores of zeolites. Therefore, it is an important issue to control the properties of the MMMs on order to separate selectively hydrogen from nitrogen.

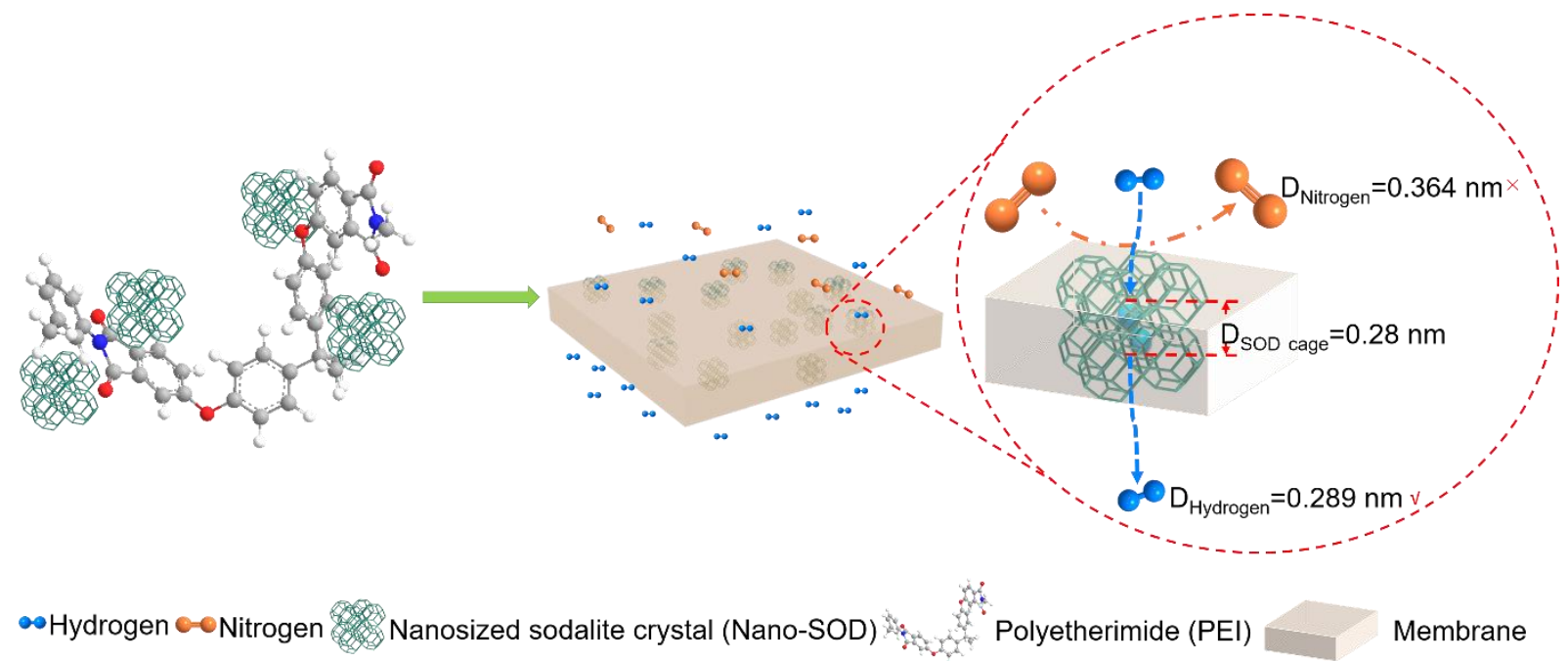

Scheme 1. Schematic presentation of mixed matrix membrane (MMMs) prepared with nanosized sodalite filler (Nano-SOD) and polyetherimide (PEI)

In this paper, we present a new method for preparation of MMMs for promoting the $\mathrm{H}_{2} / \mathrm{N}_{2}$ gas separation performance. As shown in Scheme 1, firstly, green synthesis of nanosized sodalite (SOD) crystals without organic template was performed. The nanosized sodalite crystals have uniform particle size distribution and large external surface area, which are the basis for more accessible active sites and short diffusion pathways bringing higher permeance. The nanosized sodalite crystals were highly stable and well dispersed in the polyetherimide matrix. The high molecular sieving for $\mathrm{H}_{2} / \mathrm{N}_{2}$ with sodalite crystals was achieved. The SOD with six-member rings with an aperture size of $2.8 \AA$ was chosen as filler, which only permitted small molecules like hydrogen to enter. ${ }^{[28,29]}$ Wang et al. studied the performance of sodalite-polyimide membranes in 
hydrogen and nitrogen separation. ${ }^{[30]}$ The results showed an improvement in the ideal selectivity, while the permeability of hydrogen did not change significantly, which may be due to the influence of the modifier on sodalite crystals. The hydrogen passed through the MMMs quickly because of both the short diffusion path in nanosized sodalite crystals and the openness of permeable apertures in MMMs' inner structure. While nitrogen permeation was restricted due to the molecular sieving property of nanosized sodalite. We expected the MMMs to achieve a simultaneous increase in both permeance and selectivity by utilizing nanosized sodalite crystals with the exact aperture size.

\section{Experimental Section}

\section{Materials}

Polyetherimide (PEI) was supplied by SABIC. Colloidal silica (LUDOX HS-30) and sodium aluminate $\left(37-45 \% \mathrm{Na}_{2} \mathrm{O}\right.$ and $50-60 \% \mathrm{Al}_{2} \mathrm{O}_{3}$, Technical, anhydrous) were purchased from Sigma-Aldrich and aluminium powder (-325 mesh, $99.5 \%$, metals basis) was obtained from Alfa Aesar. Sodium metasilicate pentahydrate (95\%) and 3-Aminopropyl-(diethoxy)methylsilane (APDEMS, $97 \%$ ) were purchased from Aladdin. Sodium hydroxide (NaOH) and dichloromethane $\left(\mathrm{CH}_{2} \mathrm{Cl}_{2}, 99.5 \%\right)$ were provided from Xilong Scientific. Ammonium chioride was purchased from Sinopharm Chemical Reagent Co. Ltd.

\section{Synthesis of nanosized sodalite crystals and post-synthesis treatment}

A typical synthesis mixture with the following composition $18 \mathrm{Na}_{2} \mathrm{O}: 5 \mathrm{SiO}_{2}: 1 \mathrm{Al}_{2} \mathrm{O}_{3}: 240 \mathrm{H}_{2} \mathrm{O}$ was prepared by dropwise addition of freshly prepared homogeneous aluminate solution $(0.54 \mathrm{~g}$ aluminium powder, $5 \mathrm{~g} \mathrm{NaOH}$ and $10 \mathrm{~g} \mathrm{H}_{2} \mathrm{O}$ ) into silicate solution (10 g colloidal silica, $9.368 \mathrm{~g}$ $\mathrm{NaOH}$ and $26.2 \mathrm{~g} \mathrm{H}_{2} \mathrm{O}$ ) under vigorous stirring in an ice-water bath. Then the mixture was stirred for $24 \mathrm{~h}$ at room temperature and the resulting suspension was transferred to a polypropylene 
bottle, and heated at $60^{\circ} \mathrm{C}$ under static conditions for $48 \mathrm{~h}$. The products were washed by centrifugation $(5000 \mathrm{rpm}, 20 \mathrm{~min})$, followed by re-dispersion in distilled water; this procedure was repeated several times until the $\mathrm{pH}$ of the decanted solution was 7.0. Finally the samples were dried using a freeze dryer for $60 \mathrm{~h}$ (vacuum of $5 \mathrm{~Pa},-80^{\circ} \mathrm{C}$ ). The nanosized sodalite crystals will be further abbreviated as Nano-SOD.

Post-synthesis treatment: To further study the property of Nano-SOD, two post synthesis treatments were performed. Ammonium exchange ${ }^{[31]}$ (supplementary information 1) was carried out to study the gas separation performance of SOD in proton form (sample Nano-SOD ${ }^{\mathrm{H}+}$ ). Surface modification ${ }^{[32]}$ (supplementary information 2) by coupling agent (3-Aminopropyl-(diethoxy)methylsilane, APDEMS) was applied to investigate the formation of voids or blocking of pores at the sodalite-polymer interface. The modified sample will be further abbreviated as Nano-SOD ${ }^{\text {mod }}$ in this paper.

\section{Synthesis of micronsized sodalite crystals}

The composition of the synthesis mixture for the micronsized sodalite crystals (Micro-SOD) was $50 \mathrm{Na}_{2} \mathrm{O}: 5 \mathrm{SiO}_{2}: 1 \mathrm{Al}_{2} \mathrm{O}_{3}: 1005 \mathrm{H}_{2} \mathrm{O} \cdot{ }^{[33]}$ The aluminate solution was prepared by dissolving sodium hydroxide (4.12 $\mathrm{g}$ ) in deionized water $(23.53 \mathrm{~g})$ followed by adding sodium aluminate (0.22 g). Sodium hydroxide (5.59 g) was mixed with deionized water $(23.53 \mathrm{~g})$ and sodium metasilicate $(1.66 \mathrm{~g})$ to prepare the silicate solution. After adding aluminate solution slowly into the silicate solution under stirring at room temperature, the mixture was stirred vigorously for 0.5 $\mathrm{h}$ in order to be homogenous. The resulting mixture (gel) was poured in a polypropylene bottle, and heated at $90^{\circ} \mathrm{C}$ for $22 \mathrm{~h}$. The products were purified following the procedure described above.

\section{Preparation of Mixed Matrix Membranes (MMMs)}

Polyetherimide (PEI) particles were dried at $120^{\circ} \mathrm{C}$ for $24 \mathrm{~h}$. Then the dried PEI particles $(5$ wt. \%) were added into dichloromethane $\left(\mathrm{CH}_{2} \mathrm{Cl}_{2}\right)$, and subjected to stirring for $8 \mathrm{~h}$ until the 
solution became clear (this will be named PEI matrix). All samples, including Nano-SOD, MicroSOD, Nano-SOD ${ }^{\mathrm{H}+}$, and Nano-SOD ${ }^{\text {mod }}$, were dispersed in the PEI matrix solution under sonication for $2 \mathrm{~h}$; the solutions were stationed till all bubbles were removed. These solutions were deposited on precleaned glass plates at room temperature. Thus the mixed matrix membranes (MMMs) consisting of polyetherimide and sodalite crystals (fillers) were obtained under drying at room temperature for $24 \mathrm{~h}$, followed by heating at $60^{\circ} \mathrm{C}$ for $48 \mathrm{~h}$, and $120^{\circ} \mathrm{C}$ for $24 \mathrm{~h}$. The thickness of the MMMs was between 30 and $49 \mu \mathrm{m}$. Membranes consisting of PEI and NanoSOD with mass concentrations of $0 \%, 2 \%, 10 \%, 20 \%, 30 \%$, will be further abbreviated as 0-nanoSOD-PEI, 2-nano-SOD-PEI, 10-nano-SOD-PEI, 20-nano-SOD-PEI, and 30-nano-SOD-PEI, respectively. Membranes formed with PEI and 10 wt. \% Micro-SOD, 10 wt. \% Nano-SOD ${ }^{\mathrm{H}+}, 10$ wt. \% Nano-SOD ${ }^{\text {mod }}$, will be further abbreviated as 10-micro-SOD-PEI, 10-nano-SOD ${ }^{\mathrm{H}+}-\mathrm{PEI}$, and 10-nano-SOD ${ }^{\text {mod }}$-PEI, respectively.

\section{Characterization}

X-ray diffraction (XRD) patterns of pure sodalite samples and mixed matrix membranes were recorded using a PANalytical empyrean model $\mathrm{X}$-ray diffractometer with $\mathrm{Cu} \mathrm{K} \alpha$ radiation (all samples were run in the range $5^{\circ}$ to $75^{\circ} 2 \theta$ ). Fourier transform infrared spectroscopy (FTIR) spectra were recorded using a Bruker Vertex 70 spectrometer with an average of 64 scans in the range $400-4000 \mathrm{~cm}^{-1}$. Thermal stability of nanosized sodalite crystals was evaluated by thermogravimetric analysis (TG) using a HETZSCH QMS $403 \mathrm{C}$ analyzer $\left(40-800^{\circ} \mathrm{C}\right.$ with heating rate of $10^{\circ} \mathrm{C} \cdot \mathrm{min}^{-1}$ under air). Differential scanning calorimetry (DSC) study was performed on MMMs using a HETZSCH QMS 403C analyser. The measurement was made at a heating rate of $10{ }^{\circ} \mathrm{C} \cdot \mathrm{min}^{-1}$ in a nitrogen-filler chamber, and a first run was done from $20^{\circ} \mathrm{C}$ to $400^{\circ} \mathrm{C}$ followed by quenching and a second heating. The size and morphology of sodalite crystals and the surface and cross-section of mixed matrix membranes were measured by a JEOL JSM-7900F scanning electron microscope (SEM) and a JEOL JEM-2100UHR transmission electron microscope (TEM). The 
distribution of the sodalite crystals in the membranes was measured by EDS-SEM using JEOL-7900F SEM and Oxford Instruments X-MaxN Energy-Dispersive Spectroscopy. The hydrogen and nitrogen adsorption-desorption experiments were performed at 77K using Quantachrome autosorb iQ3 gas sorption analyzer. The external surface area was determined by the Brunauer-Emmett-Teller Method (BET).The ${ }^{13} \mathrm{C}-\mathrm{NMR}$ spectra were recorded by a Bruker AVANCE III $400 \mathrm{MHz}$ spectrometers.

\section{Mixed Matrix Membranes (MMMs): permeability test}

Single and mixed gas permeability tests of pure PEI membrane and PEI/sodalite mixed matrix membranes were evaluated for $\mathrm{H}_{2}, \mathrm{~N}_{2}, \mathrm{CO}_{2}, \mathrm{CH}_{4}$ and $\mathrm{H}_{2} / \mathrm{N}_{2}$ using a permeation measurement equipment. The membranes, cut into squares with a dimension of $3.14 \mathrm{~cm}^{2}$, were placed in a stainless steel cell; both the temperatures $\left(25^{\circ} \mathrm{C}\right.$ to $\left.100^{\circ} \mathrm{C}\right)$ and pressures (atmospheric pressure) in the cell were controlled. The membranes were perched continuously by argon (Ar) flow and either single gases $\left(\mathrm{H}_{2}\right.$ or $\left.\mathrm{N}_{2}\right)$ or gas mixtures $\left(\mathrm{H}_{2} / \mathrm{N}_{2}\right)$ were delivered. A flow meter was used to measure the gas fluctuation. The volume ration of the feed gas mixture $\left(\mathrm{H}_{2}: \mathrm{N}_{2}\right)$ was regulated from 1:1 to $3: 1$. After 3 hours of permeation, the gases which penetrated the membrane were analysed by a gas chromatograph (SHIMADZU GC-2014C).

The gas permeability coefficient was calculated with the following equation:

$$
P=\frac{10^{-10} l N i}{A \Delta p i}
$$

where $P$ (Barrer) is gas permeability coefficient, $l(\mathrm{~cm})$ is the membrane thickness, $N i$ $\left(\mathrm{cm}^{3}(\mathrm{STP}) \cdot \mathrm{s}^{-1}\right)$ is the permeate flow rate of component $\mathrm{i}, A\left(\mathrm{~m}^{2}\right)$ is the effective membrane area, $\Delta p i(\mathrm{cmHg})$ is the trans-membrane pressure drop of i. $\left(1 \mathrm{Barrer}=10^{-10} \mathrm{~cm}^{3}(\mathrm{STP}) \mathrm{cm} \cdot \mathrm{m}^{-2} \cdot \mathrm{s}^{-1} \cdot \mathrm{cmHg}^{-}\right.$ 1) 
The selectivity of membrane was evaluated by the separation factor $(\alpha)$ calculated with the following equation:

$$
\alpha_{i / j}=\frac{X i / X j}{Y i / Y j}
$$

where $i, j$ represent the two components in the gas mixtures, and $X$ and $Y$ are the mole fractions in the permeance and feed solution respectively.

The gas permeability was also calculated using the Maxwell model, which describes the gas transport in mixed matrix membranes according to the following equation: ${ }^{[34]}$

$$
\boldsymbol{P}_{i M M M}=\boldsymbol{P}_{i p}\left[\frac{P_{i s}+2 P_{i p}-2 \Phi_{i s}\left(P_{i p}-P_{i s}\right)}{P_{i s}+2 P_{i p}+\Phi_{i s}\left(P_{i p}-P_{i s}\right)}\right]
$$

where $P_{i м м M}$ is the gas permeability of penetrant $i$ in the mixed matrix membranes; $P_{i p}$ and $P_{i s}$ correspond to the gas permeability of penetrant $i$ in the polymer matrix and molecular sieve particles, respectively; $\Phi_{s}$ is the volume fraction of molecular sieve particles in the whole membrane.

\section{Results and Discussion}

\section{Properties of MMMs and Nano-SOD}

The optical photographs of mixed matrix membranes (0-nano-SOD-PEI, 10-nano-SOD-PEI and 30nano-SOD-PEI) are shown in Figure S1. The presence of sodalite crystals in the mixed matrix membranes (MMMs) is confirmed by XRD (Figure S2 and S3) and FT-IR (Figure S4) characterizations; a detailed discussion of the properties of the MMMs is provided in the SI. ${ }^{[28,35,36]}$ The distribution of Nano-SOD in the MMMs is evaluated using the cross-section SEM images and energy dispersive spectroscopy analysis (Figure S5 to S7). The pristine PEI membrane (Figure S5. a) is dense. The MMMs are continuous, and no obvious voids are observed (Figure S5. b and Figure S6) due to the high dispersion and stability of SOD nanocrystals in the PEI by adding of an amount $(\leq 10 \%)$. While, when the loading of SOD 
nanocrystals increases to $20 \%$ or $30 \%$, some big holes are clearly formed, which is due to the agglomeration of sodalite nanocrystals (Figure S5. c). The SEM-EDS mapping also reveals the homogenous sodalite distribution in sample 10-nano-SOD-PEI, as well as the agglomeration of the NanoSOD crystals in sample 30-nano-SOD-PEI. The pure SOD crystals (Nano-SOD), used as a filler for the MMMs, have a mean size of 40-50 nm, which is exhibited in SEM and TEM images (Figure 1). The crystal size is also calculated by the Scherrer equation, using the two most intense Bragg peaks at $14.1^{\circ}$ and $24.5^{\circ}$ 2Theta (Figure S2. b). The average size of the sodalite crystals is $36 \mathrm{~nm}$, which is consistent with the size determined by TEM and SEM (see Figure 1). The pure Nano-SOD crystals are subjected to $\mathrm{H}_{2}$ and $\mathrm{N}_{2}$ adsorption. As shown in Figure S8, the nanosized sodalite crystals exhibit high adsorption capacity towards hydrogen, while no nitrogen was adsorbed. The nanosized sodalite crystals have higher external surface area of $\left(120.0 \mathrm{~m}^{2} \cdot \mathrm{g}^{-1}\right)$ than the micronsized sodalite $\left(2.93 \mathrm{~m}^{2} \cdot \mathrm{g}^{-1}\right)$ (Figure S9). This suggests more accessible sites for hydrogen which is beneficial for enhancing the diffusion of hydrogen in the hybrid membranes.
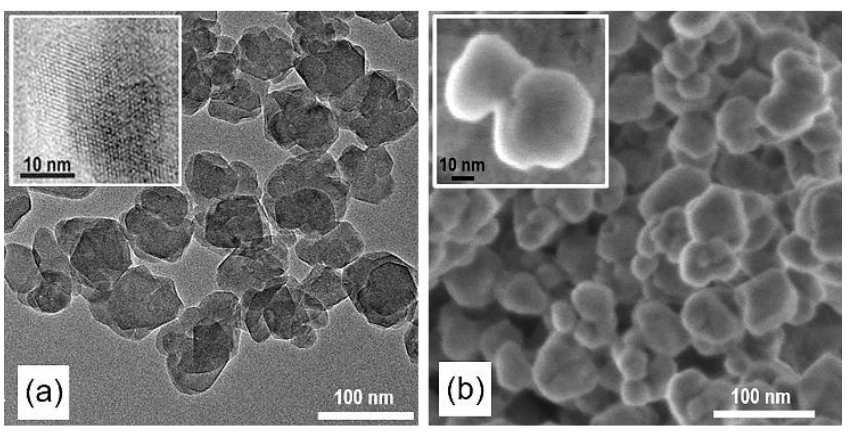

Figure 1. (a) TEM and (b) SEM images of nanosized sodalite crystals

The possible interaction between Nano-SOD and PEI was investigated by FT-IR spectroscopy and ${ }^{13} \mathrm{C}$ NMR spectroscopy. Compared with the pure Nano-SOD, the sample 10-nano-SOD-PEI showed a slight shift of the band (attribute to the deformation vibrations of the O-T-O) to lower wavenumber from 461 $\mathrm{cm}^{-1}$ to $459 \mathrm{~cm}^{-1}$ (Figure $\mathrm{S} 10$ ), suggesting the weak interaction (possibly hydrogen bonding) between the PEI and the Nano-SOD. This shift is observed in all MMMs spectra (Figure S4), illustrating that the 
highly dispersed SOD nanocrystals interact with the polymer matrix. The interaction was also studied by ${ }^{13} \mathrm{C}$ NMR spectroscopy; the spectra of pure PEI and 10-nano-SOD-PEI were recorded (Figure S11). The two peaks at $\sim 167 \mathrm{ppm}$ and $\sim 153 \mathrm{ppm}$ corresponding to $\mathrm{C} 1$ and $\mathrm{C} 2$ are present in the spectra. ${ }^{[37]}$ These two peaks shifted to a lower field as highlighted by the arrows, pointing out the weak interaction (possibly hydrogen bonding) between the polymeric PEI and the SOD nanocrystals. ${ }^{[38]}$ The high dispersion of the nanosized sodalite crystals in the polymer matrix strengthens the interaction between the PEI and sodalite, which is causing the formation of small-sized interfacial voids. The addition of aggregated sodalite crystals in the 30-nano-SOD-PEI membrane, leaded to the formation larger voids which was expected.

\section{Gas separation performance}

Following the successful preparation of the PEI/Nano-SOD MMMs, gas separation studies were performed. The PEI/Nano-SOD MMMs were mounted in a gas separation cell (Figure S12); single and mixed gases were delivered to the MMMs. The influence of the Nano-SOD loading in the MMMs on the gas separation was studied (Figure 2). As shown, the permeability for $\mathrm{H}_{2}$ increased with the increase of the amount of Nano-SOD loaded into PEI. Interestingly, when the amount of Nano-SOD reached 30 wt. \%, the permeability of hydrogen dropped sharply. The MMM doped with $10 \%$ Nano-SOD (10-nanoSOD-PEI) exhibits the best gas separation performance with the highest ideal selectivity factor $(\sim 16.9)$ and $\mathrm{H}_{2}$ permeability ( 7155.1 Barrer). In contrast, the pure PEI membrane (0-nano-SOD-PEI) has $\sim 13.5$ Barrer hydrogen permeability and 4.33 ideal selectivity index. The high $\mathrm{H}_{2}$ separation performance of the 10-nano-SOD-PEI membrane is primarily due to the presence of highly dispersed SOD nanocrystals with

uniform size (cages size of $2.8 \AA$ ) that allows only hydrogen to penetrate, while the nitrogen is rejected. Compared to 10-nano-SOD-PEI, the 30-nano-SOD-PEI has poor gas separation performance due to the significant agglomeration of Nano-SOD crystals observed by SEM and EDS-SEM. The agglomeration leads to the inefficiency of molecular sieve by blocking the active cages. 


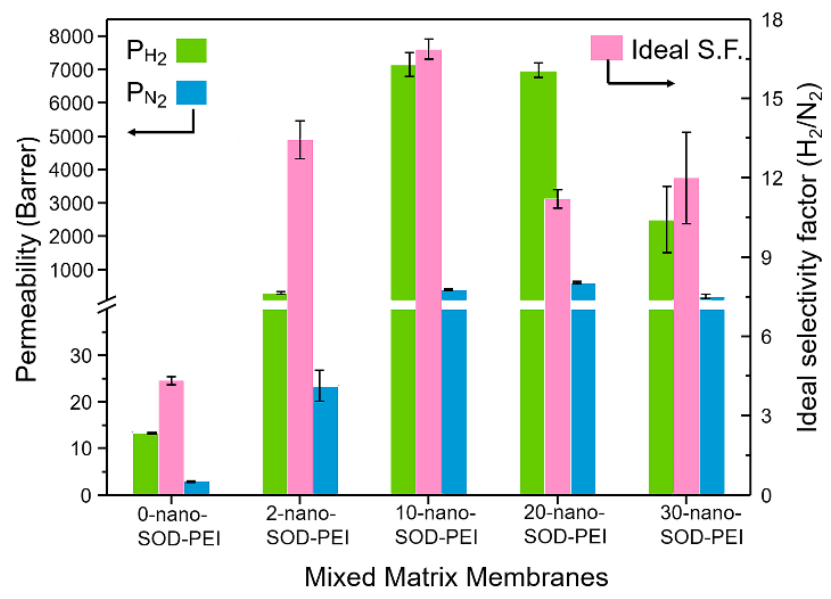

Figure 2. Single gas permeability $\left(\mathrm{P}_{\mathrm{H} 2}\right.$ and $\mathrm{P}_{\mathrm{N} 2}$ ) and ideal selectivity factor (Ideal S.F.) of MMMs at $25^{\circ} \mathrm{C}$ and atmospheric pressure

The SOD crystals in proton form are fully amorphous (see experimental section, Nano-SOD ${ }^{\mathrm{H}+}$ ) and they were used as inactive filler to prove that the hydrogen is transported through the sodalite crystals. The Nano-SOD ${ }^{\mathrm{H}+}$ sample is amorphous as seen in Figure S13. b, i.e., the diffraction peaks disappear indicating the collapse of the crystalline structure. From the TEM pictures (Figure S13. c, d), one can see that the crystal size of Nano-SOD does not change after ammonium exchange. As shown in the Figure 2, compared to the 10-nano-SOD-PEI (crystalline), the hydrogen permeability of 10 -nano-SOD ${ }^{\mathrm{H}+}-\mathrm{PEI}$ (amorphous) decreased sharply, as well as the ideal selectivity factor, chiefly attributing to the loss of crystallinity of SOD particles (collapse of the SOD cages) in the Nano-SOD ${ }^{\mathrm{H}+}$. The decrease of nitrogen permeability is related to the small voids formed between the amorphous particles $\left(\mathrm{Nano}-\mathrm{SOD}^{\mathrm{H}+}\right)$ and PEI (Figure S14). Thus it is conformed that the crystalline Nano-SOD with the appropriate aperture size is responsible for the molecule sieving of hydrogen from nitrogen. 


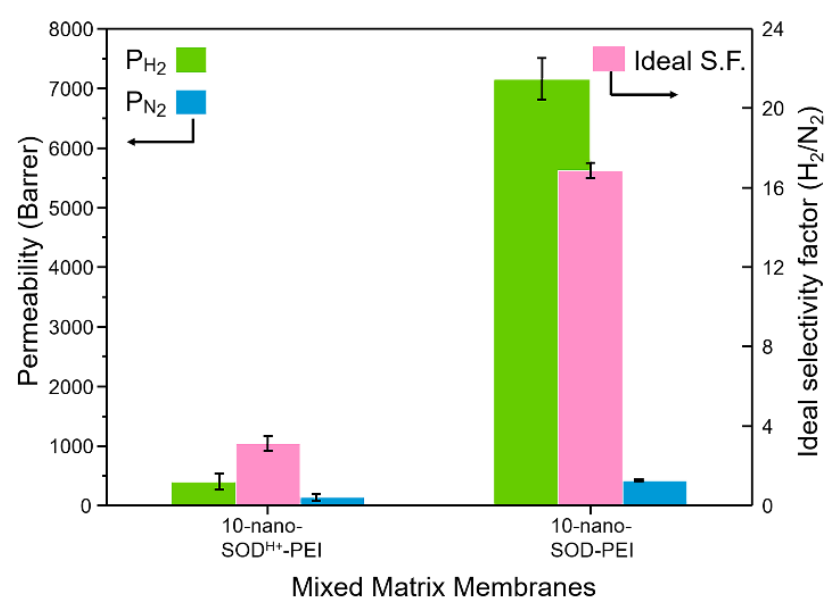

Figure 3. Performance (permeability and selectivity) of 10-nano-SOD-PEI and 10-nano-SOD ${ }^{\mathrm{H}+}$-PEI MMMs in gas separation

Table 1. The effect of small-sized interfacial voids of the membranes on gas separation.

\begin{tabular}{cccc}
\hline \multirow{2}{*}{ Membrane } & \multicolumn{2}{c}{$\mathrm{H}_{2}$ permeability (Barrer) } & $\mathrm{N}_{2}$ permeability (Barrer) \\
\cline { 2 - 4 } & Nano-SOD & Voids+PEI & Voids+PEI \\
\hline 0-nano- & 0 & $13.5 \pm 0.1$ & $3.1 \pm 0.1$ \\
SOD-PEI & & & \\
\hline 10-nano- & & \\
SOD ${ }^{\mathrm{H}+}-$ & 0 & $402.5 \pm 134.9$ & $133 \pm 62.9$ \\
PEI & & \\
\hline 10-nano- & $5875 \pm 353.8$ & $\sim 1280(18 \%)$ & $423 \pm 12.4$ \\
SOD-PEI & $(82 \%)$ &
\end{tabular}

Notes: The nitrogen permeability comes from the voids and PE. The voids in the 10-nano-SOD-PEI are bigger than in the 10 -nano-SODH ${ }^{+}$-PEI membrane; the relative ratio of $423 / 133=3.18$ is calculated. The hydrogen permeability comes from the voids and PEI. For the 10-nano-SOD-PEI membrane, the $\mathrm{H}_{2}$ permeability is calculated as follow: $402.5 \times 3.18=1280$.

The effect of small-sized interfacial voids on gas separation results of the membranes was also studied (Table 1). The increase of gas permeability of the 10-nano-SOD ${ }^{\mathrm{H}+}-\mathrm{PEI}$ membrane without the assistant 
of open-framework structure of Nano-SOD is primarily attributed to the presence of interfacial voids. Furthermore, the low gas separation index $\left(\mathrm{H}_{2} / \mathrm{N}_{2}=3.07\right)$ confirms that the process is controlled by Knudsen diffusion caused by the presence of small-sized interfacial gaps. The contribution of sodalite crystals to the hydrogen permeability is substantial (5875 Barrer). Over $80 \%$ of the total flux for the 10nano-SOD-PEI membrane is dominated by the nanosized sodalite crystals (Table 1). With the aim of further studying the effect of interfacial voids, coupling agents were used (see experimental section, Nano-SOD ${ }^{\text {mod }}$ ). Herein, the morphology of the Nano-SOD ${ }^{\text {mod }}$ is presented in Figure S15. The gas separation of this modified membrane is shown in Figure 4, exhibiting the sharp decrease of gas permeability and a small improvement in selectivity. In order to investigate the influence of the coupling agent on the permeability, the cross-section SEM images were measured and displayed in Figure S16. The severe agglomeration of sodalite crystals is observed, due to the adhesion increased by the coupling agent. As mentioned above, the high permeability is based on the sodalite nanocrystals high dispersion in the membrane. The modifier abolished the uniform dispersion of the sodalite nanoparticles, thus leading to dramatic reduction in their permeation efficiency and loss of permeability. Therefore, the modification is not suitable for the membranes in this work, as it destroys the essential advantage of the active NanoSOD filler (high permeability).

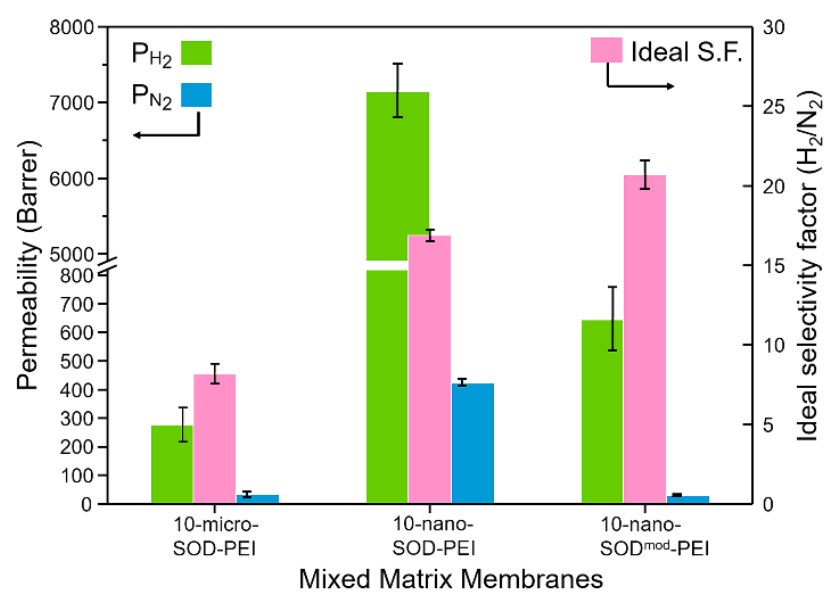

Figure 4. Performance (permeability and selectivity) of 10-nano-SOD-PEI, 10-micro-SOD-PEI and 10-

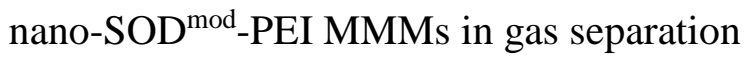


The advantages of nanosized sodalite were demonstrated through contrasting with micronsized sodalite (Figure S17). The membranes with 10 wt. \% nanosized sodalite (Nano-SOD) showed higher $\mathrm{H}_{2}$ permeability and permselectivity than the membranes prepared with micronsized counterpart (MicroSOD) (Figure 4). This is explained by the more accessible sites (due to the larger external surface area), and shorter diffusion pathway (due to the smaller crystal size) of the nanosized SOD in comparison to the micronsized SOD crystals. In the 10-nano-SOD-PEI membrane, hydrogen molecules penetrated easily in the permeable SOD apertures following the low diffusion resistance. While, the nitrogen permeation was restricted due to the molecular sieving property of the SOD crystals.

To further study the properties of the 10-nano-SOD-PEI membrane, the permeability tests for $\mathrm{CH}_{4}$ and $\mathrm{CO}_{2}$ were performed (Figure 5). The gas molecules with bigger kinetic diameter than $0.3 \mathrm{~nm}$ have apparently lower permeability than hydrogen. The permeability follows the following trend of $\mathrm{CH}_{4}>\mathrm{N}_{2}>$ $\mathrm{CO}_{2}$, thus revealing that the permeability is based on Knudsen diffusion. This suggests the existence of voids, which is consistent with the previous analysis of interfacial voids.

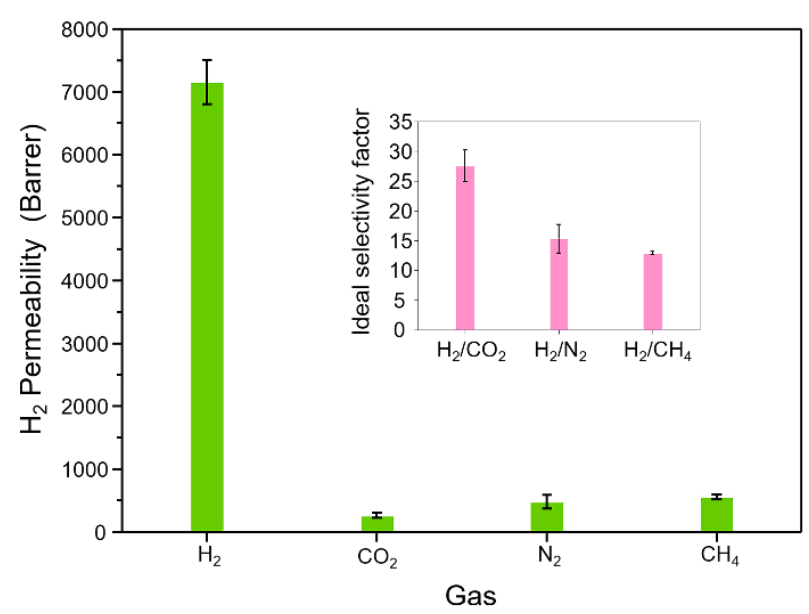

Figure 5. Single gas permeability tests of 10 -nano-SOD-PEI membrane at $25^{\circ} \mathrm{C}$

The schematic presentations of the MMMs and the potential gas transport pathways are shown in Figure 6. The possible permeation pathways in the MMMs consisting of small voids and fillers (sodalite 
and amorphous particles) could be expressed as follow: (1) polymer matrix without any fillers $\left(t_{m} / P_{m}\right)$, (2) polymer matrix containing permeable fillers with voids $\left(t_{m} / P_{m}, t_{f} / P_{f}\right.$, and $\left.t_{v} / P_{v}\right)$ and (3) polymer matrix containing impermeable fillers with voids $\left(t_{m} / P_{m}\right.$ and $\left.t_{v} / P_{v}\right) \cdot{ }^{[39]}$ Herein, the $t / P$ ratio between the transport path length and the permeability is considered as the transport resistance.

In the membranes with dispersed sodalite crystals containing voids (Figure 6. a), the permeability for hydrogen is larger than in the pure polymer permeability. Therefore, $t_{f} / P_{f}+t_{v} / P_{v}$ is much lower than $t_{m} / P_{m}$. Thus, the minimum total resistance is dominated by the area with fillers (sodalite crystals). While in the membranes with aggregated sodalite crystals (Figure 6. b), some of the apertures are blocked and the voids are connected resulting in increased resistance $t_{f} / P_{f}$. The increased resistance lead to the negative effect on the selectivity of the membrane as well. Additionally, as most zeolites are agglomerated, the proportion of the areas without filler increases, leading to decline of permeability. While the presence of amorphous particles leads to a decrease of the $P_{f}$. So the membranes containing amorphous particles display lower hydrogen permeability and selectivity (Figure 6. c).

Additionally, to minimize the overall resistance, fillers with short transport path length $\left(t_{f}\right)$ and high gas permeability $\left(P_{f}\right)$ have to be applied. The results explained the distinct difference in permeability between the membranes prepared with nanosized (Nano-SOD) and micronsized (Micro-SOD) sodalite fillers, as shown in Figure 4. In the nanosized SOD crystals, the diffusion of hydrogen is faster. ${ }^{[40]}$ While, in the micronsized sodalite crystals, not all cages are accessible and the diffusion is slower and even some cages are fully blocked.

For big molecules such as $\mathrm{N}_{2}, \mathrm{CO}_{2}$ and $\mathrm{CH}_{4}$, the sodalite cages are not accessible. The minimum total resistance is controlled by the interfacial voids. As the sodalite crystals are disperse well in the 10-nanoSOD-PEI membrane, the voids are separated and not connected. The selectivity of the membranes towards hydrogen in the $\mathrm{H}_{2} / \mathrm{N}_{2}, \mathrm{H}_{2} / \mathrm{CO}_{2}$ and $\mathrm{H}_{2} / \mathrm{CH}_{4}$ systems still maintains much higher than the Knudsen selectivity due to the molecular sieve effect of the nanosized sodalite crystals. 
The Maxwell model is used to evaluate the $\mathrm{H}_{2} / \mathrm{N}_{2}$ gas separation performance of the PEI/Nano-SOD mixed matrix membrane. The experimental and theoretical results for permeability of $\mathrm{H}_{2}$ and $\mathrm{N}_{2}$ are summarized in Table S2. As shown, the experimental values are higher than those predicted by the Maxell model. The discrepancies may be primarily attributed to the low permeability of referenced pure sodalite membrane, which is mainly limited by the size of the sodalite crystals with distinctly longer diffusion path and bigger diffusion resistance. The small size voids also influenced the precision of this ideal gas separation model.

(a)
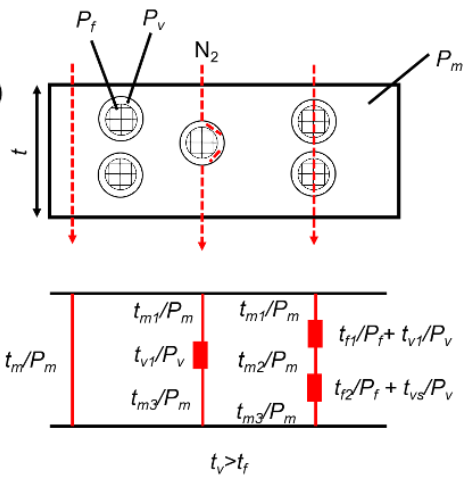

10-nano-SOD-PEI (b)
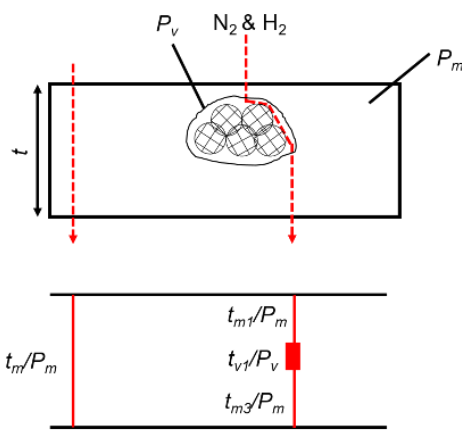

30-nano-SOD-PEI (c)
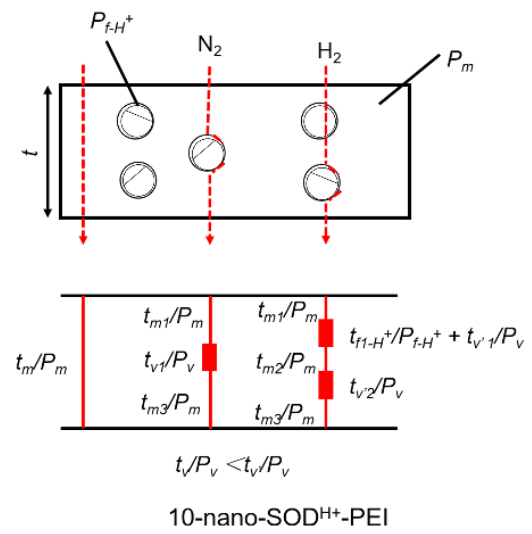

Figure 6. Schematic presentation of (a) 10-nano-SOD-PEI, (b) 30-nano-SOD-PEI, and (c) 10-nano$\mathrm{SOD}^{\mathrm{H}+}+\mathrm{PEI}$ membranes

In addition to the single gas separation experiments, mixed component $\left(\mathrm{H}_{2} / \mathrm{N}_{2}\right)$ separation test was carried out on the 10-nano-SOD-PEI membrane. The permeation and separation factors are summarized in Table S1. The permeation flux of $\mathrm{H}_{2}\left(\sim 5000\right.$ Barrer) was much higher than those of $\mathrm{N}_{2}$ in the mixture showing that the membrane had higher size selectivity preference for $\mathrm{H}_{2}$. In comparison to the results from single gas separation test ( 16.9 ideal separation factor), in the mixed component experiment a higher $\mathrm{H}_{2} / \mathrm{N}_{2}$ separation index ( 28.5) was achieved. This is due to the preferential adsorption of hydrogen thus preventing nitrogen from passing through the SOD cages. Therefore, with the increase of $\mathrm{H}_{2} / \mathrm{N}_{2}$ ratio, the separation factors for $\mathrm{H}_{2} / \mathrm{N}_{2}$ is increased sharply. As shown in Figure 7, the $\mathrm{H}_{2}$ permeation increased, while the $\mathrm{N}_{2}$ permeation decreased when the feed gas ratio increased. An experiment with a $\mathrm{H}_{2} / \mathrm{N}_{2}$ 
proportion of 3 was performed, which is similar to the condition of simulated ammonia production. The 10-nano-SOD-PEI membrane possessed both unusual high separation index $(\sim 380)$ and permeance ( 7700 Barrer), which would be of great significance for further industrial application.

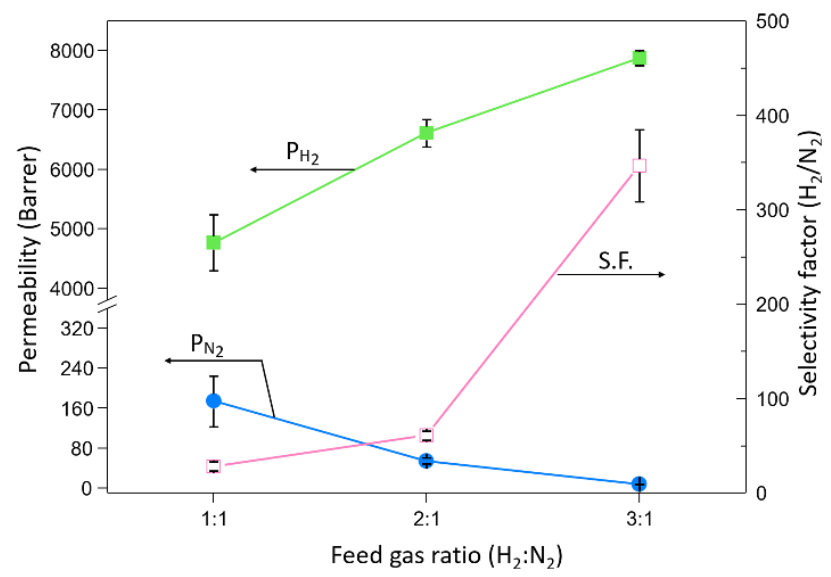

Figure 7. Influence of feed gas ratio $\left(\mathrm{H}_{2} / \mathrm{N}_{2}\right)$ on gas separation performance of 10-nano-SOD-PEI membrane

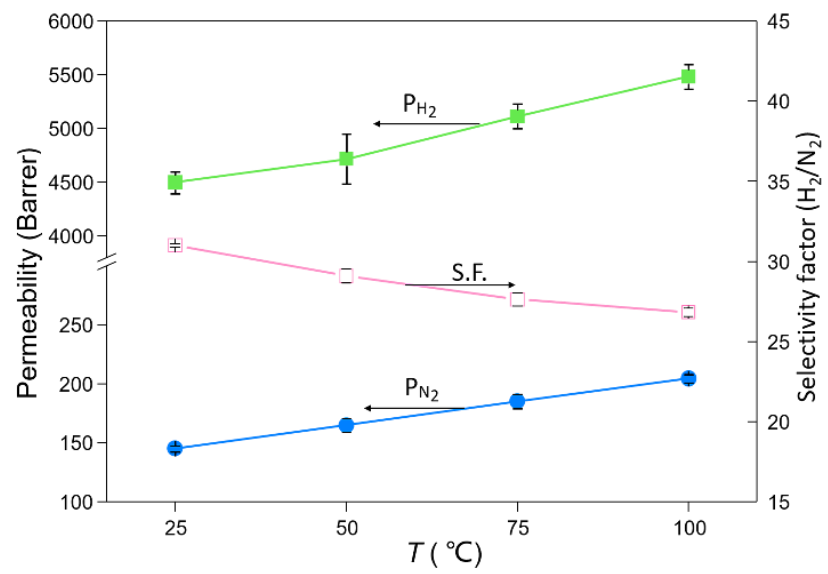

Figure 8. Permeability and separation of 10-nano-SOD-PEI membrane at different temperatures (25$\left.100^{\circ} \mathrm{C}\right)$

In order to assess the thermal stability of the 10-nano-SOD-PEI, the DSC curve and the pictures of the membrane at three temperatures are shown in Figure S18. As shown, the glass transition temperature $(\mathrm{Tg})$ was above $215^{\circ} \mathrm{C}$, which is in accordance with other reports. ${ }^{[41]}$ The variation of the temperature 
$\left(25^{\circ} \mathrm{C}-100^{\circ} \mathrm{C}\right)$ on the performance of the 10 -nano-SOD-PEI was measured. The $\mathrm{H}_{2}$ permeation flux increased while the selectivity remains unchanged (Figure 8). These results demonstrate the great advantage of the MMMs containing SOD nanocrystals especially for the gas separation processes carried out at high temperature. The reproducibility and durability of the membrane were also examined. As shown in Figure 9, the separation factors and permeations over a 6-week period remained constant for the 10-nano-SOD-PEI membrane. This membrane could be used continuously over a long period of time proving the significant stability and recyclability; the 10-nano-SOD-PEI membrane was subjected to test for 6 months continuously. Besides, the same membrane was tested at different temperatures $\left(25-100^{\circ} \mathrm{C}\right)$ after continuous 3-weeks experiments. An increase in $\mathrm{H}_{2}$ permeability was observed due to the expansion of polymer after high temperature treatment. The membrane shows the same high selectivity. No differences of the membrane's before and after temperature tests are seen in the SEM images (Figure S19) demonstrating the high stability.

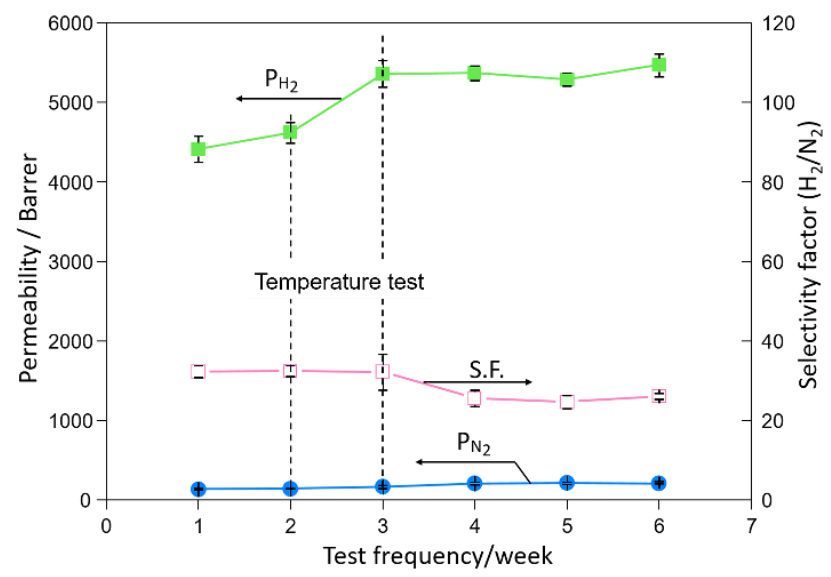

Figure 9. Reproducibility and durability of 10-nano-SOD-PEI membrane for $\mathrm{H}_{2} / \mathrm{N}_{2}$ gas separation for 6 weeks continuously

The MMM consisting of PEI solution with $10 \%$ nanosized SOD crystals reveals great improvement in gas separation. As shown in Figure 10, the MMM prepared in this work exhibits the highest $\mathrm{H}_{2}$ permeability (10-100 times higher), compared with other mixed matrix membranes. Although it shows 
slightly less selectivity than other mixed matrix membranes, it is important to note that the current MMM was not subjected to any modification. Additionally the thickness of the membrane could be further optimized. The MMM presented here reveals high hydrogen permeability similar to MOF and zeolites, while a higher selectivity at the same time (Figure S20). Besides the high gas separation performance, the high stability and easy processing make the membranes suitable for industrial application and more specifically for synthetic ammonia that requires vast hydrogen, and also releases large amounts of hydrogen.

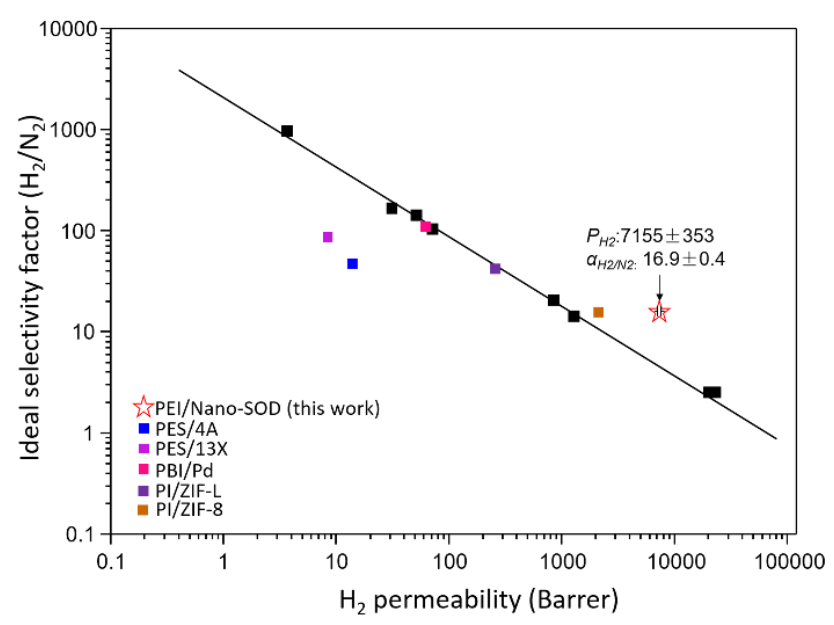

Figure 10. $\mathrm{H}_{2} / \mathrm{N}_{2}$ gases separation performance of 10-nano-SOD-PEI membrane compared with literature data $\left(\mathrm{PES} / 4 \mathrm{~A},{ }^{[20]} \mathrm{PES} / 13 \mathrm{X},{ }^{[20]} \mathrm{PBI} / \mathrm{Pd},{ }^{[42]} \mathrm{PI} / \mathrm{ZIF}-\mathrm{L},{ }^{[43]} \mathrm{PI} / \mathrm{ZIF}-8^{[43]}\right)$ using Robeson's upper bound

\section{Conclusions}

In the present study, we used nanosized, homogeneously dispersed sodalite crystals as filler in the preparation of mixed matrix membranes. The membranes show high $\mathrm{H}_{2}$ permeability and $\mathrm{H}_{2} / \mathrm{N}_{2}$ selectivity. This membrane will be of great interest especially in synthetic ammonia purge gas purification.

The advantages of using SOD nanocrystals for the MMMs are: (1) proper aperture size of sodalite cages allowing only hydrogen to penetrate while nitrogen to is rejected; (2) nanosized sodalite crystals 
have large external surface area and shorter diffusion pathways originated from the small particle size (40 $\mathrm{nm}$ ); (3) highly stable nanosized sodalite crystals in polymer matrix leading to the maximum utilization of their unique properties and preventing cage blocking and agglomeration. Finally, the mixed matrix membranes with good continuity, high stability and reproducibility demonstrated high $\mathrm{H}_{2}$ flux and selectivity.

\section{Acknowledgements}

The authors gratefully acknowledged funding from Thousand Talents Program for Foreign Experts (WQ20152100284), the French-China Science Foundation (FFCSA), the National Natural Science Foundation of China (Grant No. 21673291), and the State Key Laboratory of Advanced Technology for Materials Synthesis and Processing (Wuhan University of Technology) (No. 2018-KF-13) and the International Associated Laboratory (LIA)-Zeolites.

\section{References}

[1] S. Giddey, S. P. S. Badwal, A. Kulkarni, Int. J. Hydrogen Energ. 2013, 38, 14576-14594.

[2] V. Kyriakou, I. Garagounis, E. Vasileiou, A. Vourros, M. Stoukides, Catal. Today. 2017, 286, 2-13.

[3] B. T. Low, N. Widjojo, T. S. Chung, Ind. Eng. Chem. Res. 2010, 49, 8778-8786.

[4] C. G. D. A. RAAB, Mol. Phys. 1998, 93, 49-56.

[5] Q. Sun, M. Wang, Z. Li, Y. Ma, A. Du, Chem. Phys. Lett. 2013, 575, 59-66.

[6] G. A. Prody, J. T. Bakos, J. M. Buzayan, I. R. Schneider, G. Bruening, Science. 1986, 231, $1577-$ 1580.

[7] A. S. Damle, S. K. Gangwal, V. K. Venkataraman, Gas Separation \& Purification. 1994, 8, 101-106.

[8] D. LEE, S. PARK, C. YU, S. IHM, K. LEE, J. Membrane Sci. 2007, 302, 265-270.

[9] C. Song, Q. Liu, N. Ji, Y. Kansha, A. Tsutsumi, Appl. Energ. 2015, 154, 392-401.

[10] S. Adhikari, S. Fernando, Ind. Eng. Chem. Res. 2006, 45, 875-881. 
[11]H. Chen, Y. Xiao, T. Chung, Polymer. 2010, 51, 4077-4086.

[12] S. Kim, E. Shamsaei, X. Lin, Y. Hu, G. P. Simon, J. G. Seong, J. S. Kim, W. H. Lee, Y. M. Lee, H. Wang, J. Membrane Sci. 2018, 549, 260-266.

[13]L. M. Robeson, J. Membrane Sci. 2008, 320, 390-400.

[14]F. Gallucci, E. Fernandez, P. Corengia, M. van Sint Annaland, Chem. Eng. Sci. 2013, 92, 40-66.

[15] S. M. Lee, N. Xu, J. R. Grace, A. Li, C. J. Lim, S. S. Kim, F. Fotovat, A. Schaadt, R. J. White, J. Eur. Ceram. Soc. 2018, 38, 211-219.

[16] M. S. Nabavi, T. Mohammadi, M. Kazemimoghadam, Ceram. Int. 2014, 40, 5889-5896.

[17]P. Li, Z. Wang, Z. Qiao, Y. Liu, X. Cao, W. Li, J. Wang, S. Wang, J. Membrane Sci. 2015, 495, 130168.

[18]D. Bastani, N. Esmaeili, M. Asadollahi, J. Ind. Eng. Chem. 2013, 19, 375-393.

[19]H. Vinh-Thang, S. Kaliaguine, Chem. Rev. 2013, 113, 4980-5028.

[20] M. G. Süer, N. Baç, L. Yilmaz, J. Membrane Sci. 1994, 91, 77-86.

[21]C. I. Chaidou, G. Pantoleontos, D. E. Koutsonikolas, S. P. Kaldis, G. P. Sakellaropoulos, Sep. Sci.Technol. 2012, 47, 950-962.

[22] M. Peydayesh, T. Mohammadi, O. Bakhtiari, Energy. 2017, 141, 2100-2107.

[23]H. H. Yong, H. C. Park, Y. S. Kang, J. Won, W. N. Kim, J. Membrane Sci. 2001, 188, 151-163.

[24] M. Rezakazemi, K. Shahidi, T. Mohammadi, Int. J. Hydrogen Energ. 2012, 37, 14576-14589.

[25] J. M. Duval, A. J. B. Kemperman, A. Folkers, M. H. V. Mulder, G. Desgrandchamps, C. A. Smolders, J. Appl. Polym. Sci. 1994, 1994, 409-418.

[26]H. Tseng, H. Chuang, G. Zhuang, W. Lai, M. Wey, Int. J. Hydrogen Energ. 2017, 42, 11379-11391.

[27]A. L. Khan, A. Cano-Odena, B. Gutiérrez, C. Minguillón, I. F. J. Vankelecom, J. Membrane Sci. 2010, 350, 340-346.

[28] W. Fan, K. Morozumi, R. Kimura, T. Yokoi, T. Okubo, Langmuir. 2008, 24, 6952-6958.

[29]X. Xu, Y. Bao, C. Song, W. Yang, J. Liu, L. Lin, Micropor. Mesopor. Mat. 2004, 75, 173-181. 
[30] D. Li, H. Y. Zhu, K. R. Ratinac, S. P. Ringer, H. Wang, Micropor. Mesopor. Mat. 2009, 126, 1419.

[31]H. Awala, J. Gilson, R. Retoux, P. Boullay, J. Goupil, V. Valtchev, S. Mintova, Nat. Mater. 2015, $14,447-451$.

[32] A. Ebadi Amooghin, M. Omidkhah, A. Kargari, J. Membrane Sci. 2015, 490, 364-379.

[33] S. Khajavi, F. Kapteijn, J. C. Jansen, J. Membrane Sci. 2007, 299, 63-72.

[34] G. Liu, V. Chernikova, Y. Liu, K. Zhang, Y. Belmabkhout, O. Shekhah, C. Zhang, S. Yi, M. Eddaoudi, W. J. Koros, Nat. Mater. 2018, 17, 283-289.

[35]M. E. Brenchley, M. T. Weller, Journal of Materials Chemistry. 1992, 2, 1003-1005.

[36]D. Novembre, B. DI. Sabatino, D. Gimeno, C. Pace, Clay Miner. 2011, 46, 339-354.

[37]D. M. White, T. Takekoshi, F. J. Williams, H. M. Relles, P. E. Donahue, H. J. Klopfer, G. R. Loucks, J. S. Manello, R. O. Matthews, R. W. Schluenz, J. Polym. Sci. Pol. Chem. 1981, 19, 1635-1658.

[38]E. Leite, I. Naydenova, S. Mintova, L. Leclercq, V. Toal, Appl.Optics. 2010, 49, 3652.

[39]H. Vinh-Thang, S. Kaliaguine, Chem. Rev. 2013, 113, 4980-5028.

[40] A. W. C. van den Berg, S. T. Bromley, E. Flikkema, J. C. Jansen, J. Chem. Phys. 2004, 121, 1020910216.

[41]T. Liu, R. Ozisik, R. W. Siegel, Thin Solid Films. 2007, 515, 2965-2973.

[42]H. S. M. Suhaimi, C. P. Leo, A. L. Ahmad, Chem. Eng. Technol. 2017, 40, 631-638.

[43] S. Kim, E. Shamsaei, X. Lin, Y. Hu, G. P. Simon, J. G. Seong, J. S. Kim, W. H. Lee, Y. M. Lee, H. Wang, J. Membrane Sci. 2018, 549, 260-266. 
Mixed matrix membranes with uniformly dispersed nanosized sodalite crystals in polymeric matrix are prepared. The sodalite nanocrystals are synthesized free of organic template with large external surface area and monomodal particle size distribution. The sodalite nanocrystals with proper aperture size of the cages $(2.8 \AA)$ allow only $\mathrm{H}_{2}$ to penetrate while $\mathrm{N}_{2}$ is rejected. The mixed matrix membranes show high $\mathrm{H}_{2}$ flux and selectivity, and will be of great importance for synthetic ammonia processing that demands and releases a large amount of hydrogen.

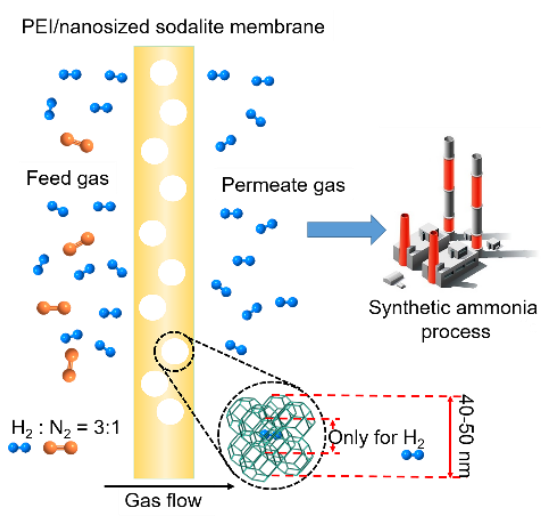

Ge Yang, Hailing Guo*, Zixi Kang, Lei Zhao, Shou Feng, Feng Jiao, Svetlana Mintova*

Page No. - Page No.

Title: Green hydrogen separation from nitrogen by mixed matrix membranes consisting of nanosized sodalite crystals 